\title{
Foreword
}

\section{Anesthetic Care for Abdominal Surgery}

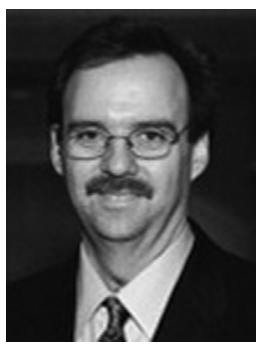

Lee A. Fleisher, MD

Consulting Editor

With the recent interest in providing value in surgical care, enhanced recovery after surgery protocols and the perioperative management of the patient undergoing colorectal surgery has taken on increasing importance. This is particularly true given the recent interest in the Perioperative Surgical Home. In addition, there have been changes in the manner in which anesthesiologists provide care for a group of other abdominal surgeries. In this issue of Anesthesiology Clinics, a remarkable group of international experts in the field has written outstanding reviews to help all of us provide state-ofthe-art value-based care.

In choosing an editor for an abdominal surgery issue, it was easy to choose two outstanding contributors to this field. Dr. Timothy Miller is currently Assistant Professor of Anesthesiology at Duke University. Dr. Michael Scott is a Consultant in Anaesthesia and Intensive Care Medicine at the Royal Surrey County Hospital NHS Foundation Trust and Senior Fellow at the University of Surrey. Given their expertise, they are well qualified to edit this important issue.

Lee A. Fleisher, MD

Perelman School of Medicine

University of Pennsylvania Philadelphia, PA 19104, USA

E-mail address:

lee.fleisher@uphs.upenn.edu 